\title{
Proximal Avulsion of Ulnar Collateral Ligament of the Thumb
}

\section{Maklad $\mathrm{M}^{*}$, Chu H, Delikonstantinou I and West E}

Southmead Hospital, Bristol, United Kingdom

${ }^{*}$ Corresponding author: Maklad M, Southmead Hospital, Bristol, United Kingdom, Tel: 00447584563730, E-mail: mohamedmaklad@doctors.org.uk

Citation: Maklad M, Chu H, Delikonstantinou I, West E (2019) Proximal Avulsion of Ulnar Collateral Ligament of the Thumb. J Case Rep Stud 7(4): 401. doi: 10.15744/2348-9820.7.401

Received Date: June 8, 2019 Accepted Date: August 26, 2019 Published Date: August 28, 2019

\begin{abstract}
Injuries of the Ulnar collateral ligament (UCL) of the metacarpophalangeal (MCP) joint of the thumb are relatively common and well described in the literature. Stener lesion refers to the displacement of the avulsed distal end of the UCL, moving to lie over the adductor aponeurosis making the chances of spontaneous healing impossible [1].

We present a case report of an Ulnar collateral ligament injury with avulsion of the proximal end of the ligament. Whilst this is referred to in text books, there are no reported individual cases or series in the published literature.

Keywords: Ulnar Collateral Ligament; UCL; Stener Lesion
\end{abstract}

\section{Case Report}

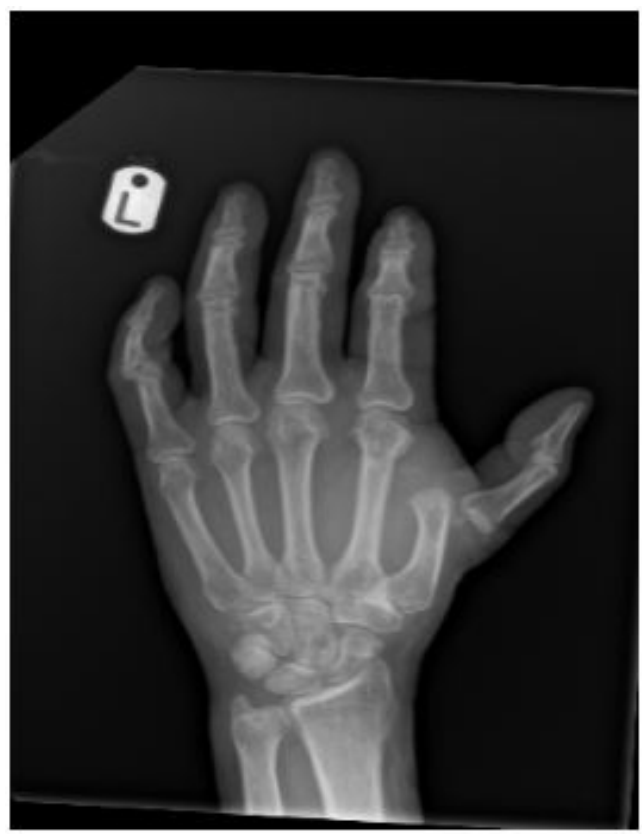

Figure 1: Dislocated thumb MCP joint

60 year old patient presented to Emergency Department after a high energy road traffic accident. He was thrown of his motorbike while driving at $60 \mathrm{mph}$. On examination, his left thumb was obviously deformed and swollen and X ray revealed a dislocated left thumb MCP joint (Figure 1). Post reduction examination revealed multiaxial instability.

He had also sustained a PIPJ fracture dislocation of left little finger and fracture of the base of right $5^{\text {th }}$ metacarpal bone of the right hand. Due to the severity of injury and multiaxial instability of the MCP joint, no further imaging modality as ultrasound or MRI was arranged.

The patient was operated under general anaesthesia. Exploration of the MCP joint revealed a torn dorsal joint capsule, volar plate and proximally avulsed Ulnar collateral ligament (Figure 2). 


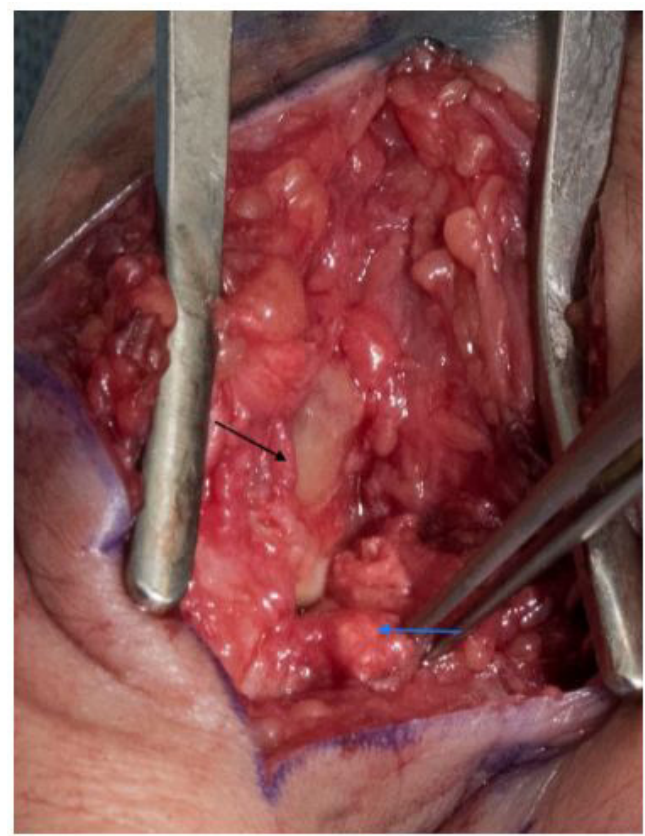

Figure 2: Blue arrow points to avulsed proximal end of the UCL. Black arrow points to divided adductor pollicis aponeurosis

The UCL was anchored to the metacarpal using mitek microanchor, the dorsal capsule was repaired, and volar plate was redraped and repaired. The MCP was stabilised using single oblique K wire (Figure 3).

The repair was protected in thumb spica for 4 weeks, at which point the wire was removed. A protective thermoplastic splint was worn for a further 2 weeks. At 10 weeks post repair, the patient achieved good stability and full mobility at the MCP and discharge from the hand therapy care.

\section{Discussion}

Complete clinical ruptures of the UCL are commonly managed surgically, as often have a Stener lesion, and the debate in the literature is whether pre-operative assessment with USS or MRI will help us to make this decision.

USS or MRI scans which confirm complete UCL tear can always be treated conservatively as these injuries don't produce Stener lesion. However, follow up with X ray at 6 weeks would be required to check the alignment of the joint.

In clinical practice, and the published literature, a proximally avulsed UCL is unreported. Whilst this may heal spontaneously, as does not have an interposing adductor aponeurosis. We believe that it will only occur with a high energy injury and, as in this case, be associated with disruption of other elements of the MCPJ stability. This therefore requires surgical repair and K wire stabilisation, and management is the same as a distally avulsed UCL.

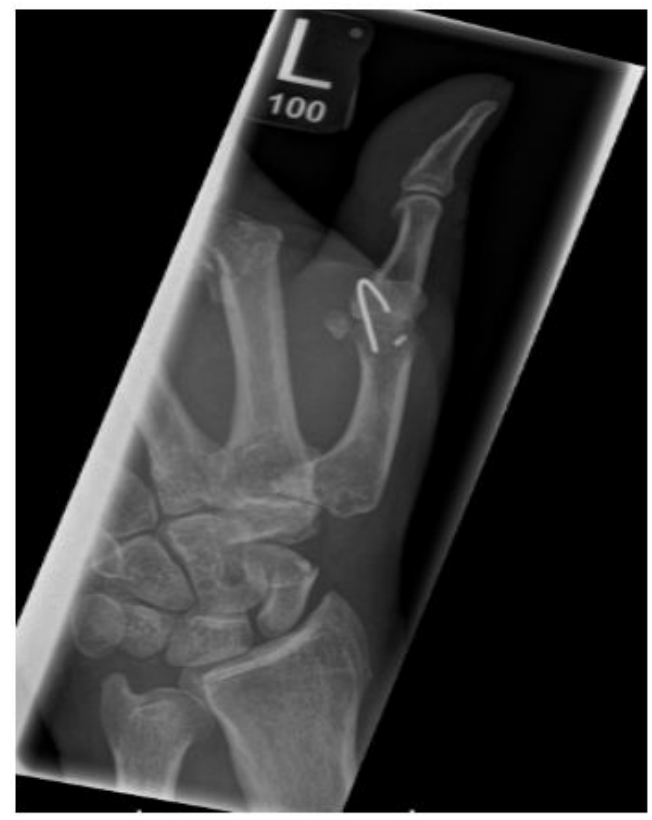

Figure 3: Post repair of the ligament and joint stabilisation 


\section{References}

1. Moberg E, Stener B (1953) Injuries to the ligaments of the thumb and fingers: diagnosis, treatment and prognosis. Acta Chir Scand 106: 166-86.

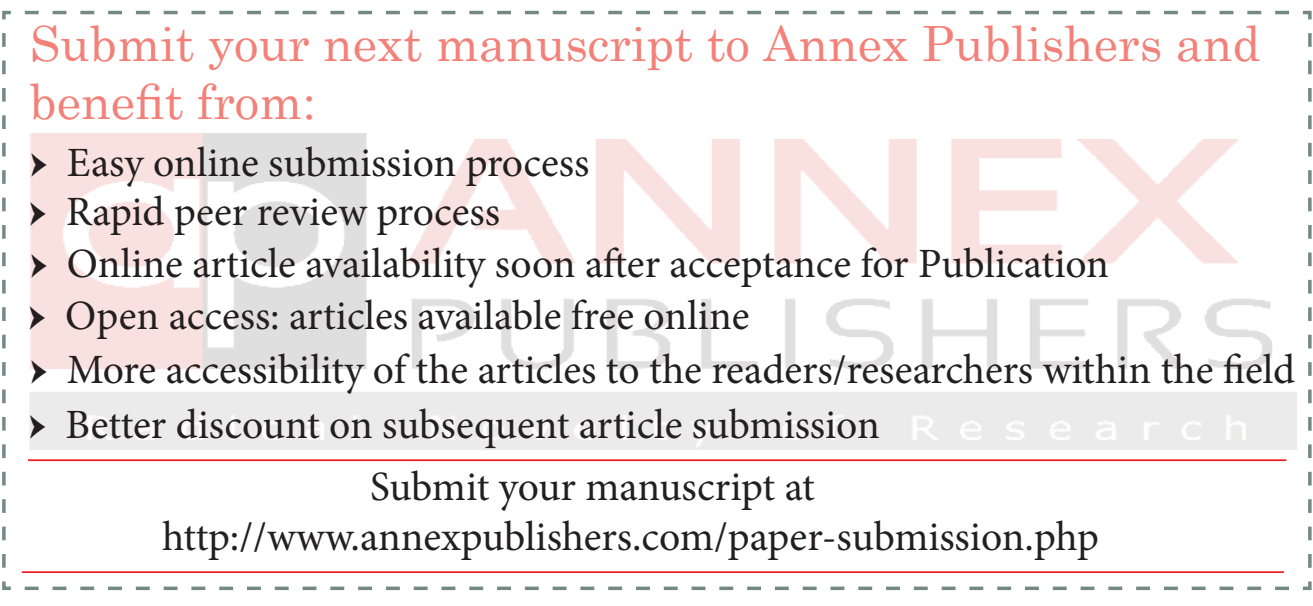

